\title{
AN IMPROVED TRANSABDOMINAL PREPERITONEAL ALLOPLASTY FOR RECURRENT INGUINAL HERNIAS AFTER LICHTENSTEIN'S SURGERY
}

\author{
Yaroslav Feleshtynskyi ${ }^{1}$ \\ Kyiv City Clinical Hospital No. 5 \\ 11 Vidpochinky str., Kyiv, Ukraine, 03115 \\ feleshtynsky@yahoo.com \\ Andrii Shtaier ${ }^{1}$ \\ Kyiv City Clinical Hospital No. 1 \\ 121 Kharkivske highway, Kyiv, Ukraine, 02096 \\ kolochava@ukr.net \\ ${ }^{1}$ Department Surgery and Proctology \\ P. L. Shupyk National Medical Academy of Postgraduate Education \\ 9 Dorohozhytska str., Kyiv, Ukraine, 04112
}

\begin{abstract}
The aim. Improve results of the surgical treatment of recurrent inguinal hernias after Lichtenstein's surgery by using an advanced TAPP technique.

Materials and methods. An analysis of the surgical treatment of patients with recurrent inguinal hernias after Lichtenstein's surgery using traditional and improved preperitoneal transabdominal alloplasty (TAPP), for the period of 2012-2019, was performed. The traditional TAPP technique was performed for 52 patients who made up the 1st group. An improved TAPP technique was implemented for 53 patients who composed the 2nd group.

The features of the improved TAPP technique, which was different from the traditional one, were by additional mobilization of the parietal peritoneum by $3-4 \mathrm{~cm}$ along the upper edge of the defect, the mesh implant was used with a larger size in comparison to the classical one $-15 \times 15 \mathrm{~cm}$ and fixed, besides the traditional points, additionally on the lower and lateral edges with medical glue Sulfacrylate.

Results. The results of surgical treatment in the early postoperative period were not significantly different and were comparable. During the long-term period, 51 patients from the 1st group, and 50 patients from the 2nd group were examined. Thus, in the first group in 4 (7.9\%) cases during 6 months period after the application of the traditional TAPP technique, chronic pain was observed on the site of the implanted mesh; among the 2 nd group of patients chronic inguinal pain was not observed. In 5 ( $9.8 \%$ ) patients of the 1st group, the recurrence of inguinal hernia was diagnosed, instead of the 2nd group, where relapse was observed in $1(2 \%)$ case.

Conclusions. Thereby, the results of the traditional and improved TAPP techniques confirm the higher efficiency of the improved technique, due to the absence of the chronic inguinal pain and a lower rate of relapses, which is achieved by wider mobilization of the parietal peritoneum along the upper edge of the defect, and usage of the larger mesh and its additional fixation by gluing it at the lower lateral edge.
\end{abstract}

Keywords: recurrent inguinal hernia, TAPP, optimization of alloplasty of recurrent hernias.

DOI: $10.21303 / 2504-5679.2020 .001364$

\section{Introduction}

Most surgical interventions for inguinal hernias are performed using Lichtenstein's surgery $[1,2]$. However, the Lichtenstein's operation is the most common, accessible by most surgeons, and relatively technically simple - the recurrence rate of inguinal hernias remains at the level of 3.1-10\% [3-5]. The treatment of recurrent inguinal hernias after Lichtenstein's surgery with Lichtenstein's reoperation is technically difficult [6], due to scarring changes in the tissues of the inguinal region and altered anatomy of the inguinal canal, accompanied with an even higher frequency of repeated recurrences of 9.3-12\% [7, 8]. Thereby, in the surgical treatment of inguinal hernias, posterior preperitoneal access is more appropriate [9]. Among the opened and laparoscopic preperitoneal techniques, according to the EHS recommendation [1], the transabdominal preperito- 
neal approach alloplasty (TAPP) is more relevant. At the same time, the traditional TAPP technique in the treatment of recurrent inguinal hernias is accompanied by repeated recurrences, which make $6-8 \%$ [10]. The main reasons for repeated recurrences of inguinal hernias after traditional TAPP approach are insufficient coverage of the hernial defect with mesh, the absence of mesh fixation along its lower edge in the projection of the iliac vessels, and trauma to the femoral nerve branches during mechanical mesh fixation $[11,12]$.

In our opinion, improvements in the traditional TAPP approach will facilitate the amelioration of the treatment outcomes for recurrent inguinal hernias after Lichtenstein's surgery.

The aim of the work. Improve results of the surgical treatment of recurrent inguinal hernias after Lichtenstein's surgery by using an advanced TAPP technique.

\section{Materials and methods}

The analysis of the surgical treatment of 105 patients with recurrent inguinal hernias after Lichtenstein's surgery was held in the clinic of the Department of Surgery and Proctology, P. L. Shupyk National Medical Academy of Postgraduate Education. All patients were male. The age of patients is from 24 to 76 years old (average age $53.4 \pm 1.2$ ). Concomitant pathology was diagnosed in $41(39.1 \%)$ cases with a predominance of chronic cardiovascular diseases in the stage of compensation.

The Ethics Commission of the Shupyk National Medical Academy of Postgraduate Education approved and authorized the study on January 10, 2012 No. 2, and confirmed that the study complies with the Helsinki Declaration of the World Medical Association. All patients who participated in the study agreed to conduct this study.

According to the classification of G. Campanelli [13], patients were distributed as follows: R1 recurrent high lateral hernias with a slight defect were observed in 36 (34.3\%) patients, R2 was low medial hernias with a slight defect in 55 (52.4\%) cases, R3 recurrent hernias with a large defect - in $14(13.3 \%)$ patients. In concern to the occurrence of relapse, in particular, 3 months after surgery, hernia recurrence occurred in $26(24.8 \%)$ cases, after 6 months in $63(60.0 \%)$ cases, and after 12 months in $16(15.2 \%)$ patients.

The traditional TAPP technique was performed during the period of 2012-2016 yrs for 52 patients with recurrent inguinal hernias after Lichtenstein's surgery, which made up the 1st group. An improved TAPP technique was performed during the period of 2017-2019 yrs for 53 patients, with recurrent inguinal hernias after Lichtenstein's surgery, which made up the 2nd group. Patient groups were comparable in patients' age and size of recurrent inguinal hernias. Antibiotic prophylaxis in patients of both groups was performed by administering a daily dose of Third generation cephalosporins (ceftriaxone 2.0) before surgery.

The traditional TAPP technique, which was performed in patients of the first group, consisted in the fact that under the general anaesthesia after application of the carboxyperitoneum, three trocars were placed using a Veress needle: $10 \mathrm{~mm}$ below the belly button, two $5 \mathrm{~mm}$ trocars at the same level along the edges of the rectus abdominis muscles. After diagnostic laparoscopy, the parietal peritoneum from the iliopubic tract to the medial umbilical fold was arcuately cut along the upper edge of the hernial defect. The mobilization of the parietal peritoneum with distinguishing of the hernial sac was performed. After that, a 12x15 cm polypropylene hernia mesh was placed preperitoneally and fixed with a hernia stapler Protac, along the upper lateral edge, to the Cooper's ligament and abdominal muscles. The peritoneum was sutured with continuous running sutures with Vicryl 2-0.

In the second group of patients, improved TAPP technique was performed [14], which was different from the traditional one by additional mobilization of the parietal peritoneum by $3-4 \mathrm{~cm}$ along the upper edge of the defect, the mesh implant was used with a larger size in comparison to the classical one $-15 \times 15 \mathrm{~cm}$ and fixed, besides the traditional points, additionally on the lower and lateral edges with medical glue Sulfacrylate $[15,16]$.

Evaluation of the results of traditional TAPP technique in the 1st group of patients and improved TAPP technique was carried out by taking into consideration complications in the early postoperative period (seroma, infection of trocar wounds, the period of recovery of the physical 
activity). In the remote postoperative period after $6,24,36$ months results were assessed by questioning, re-examination, evaluation of the presence or absence of the chronic inguinal pain [17, 18], and repeated hernia recurrence.

Descriptive statistics were generated for the baseline, immediate postoperative and longterm periods. Comparison groups were carried by $\mathrm{T}$ test (timing recovery of physical activity, the normal approximation by Shapiro-Wilk test) and the chi-square test (cases complications). Significance was set at $\mathrm{p}<0.05$.

\section{Results}

The immediate results of the performed traditional TAPP method in the 1st group of patients showed that scrotal hematoma was diagnosed in 4 (7.7 \%) patients, seromas at the site of mesh implantation in $5(9.6 \%)$ cases, there were no infections of trocar wounds. Recovery of the physical activity was after 10+2.1 days. Similar immediate results were observed among 2nd group of patients, to whom performed an improved TAPP technique. Seromas occurred in $6(11.3 \%)$ patients ( $\mathrm{p} 2-1=0.775)$, the scrotal hematoma was observed in $3(5.7 \%)$ patients $(\mathrm{p} 2-1=0.676)$, infected trocar wounds was not observed, and the physical activity was restored after $10+1.6$ days. Scrotal hematomas that were observed in patients of both groups with inguinal-scrotal hernias were associated with distinguishing of the hernial sac and were eliminated with conservative treatment. Seromas were also eliminated by conservative treatment (nonsteroidal anti-inflammatory drugs), which was confirmed by ultrasound diagnostics of the abdominal wall.

The long-term results, among 51 patients of group 1 in 4 (7.9\%) cases, during the 6 months period after the application of the traditional TAPP technique, chronic pain was observed on the site of the implanted mesh, which was periodically relieved by analgesics and nonsteroidal anti-inflammatory drugs. Among the 2nd group of patients, chronic inguinal pain was not observed.

Among 51 patients of the 1 st group who were examined by questionnaire, control examinations, and ultrasound diagnostics of the abdominal wall after 6, 12, 24, 36 months after surgery. In $5(9.8 \%)$ cases, the recurrence of inguinal hernia was diagnosed. In 2 patients, recurrence of inguinal hernia occurred 7 months after surgery, in 3 patients after 9-11 months, respectively. During the observation of 50 patients of group 2 within 6-36 months, to whom were performed improved TAPP technique, recurrence of inguinal hernia was observed in $1(2 \%)$ patient $(\mathrm{p} 2-1=0.097, \mathrm{OR}=0.188(0.004-1.79), 2$ months after surgery, as a result of excessive physical exertion 2 weeks after surgery. Evaluation of immediate and long-term results indicates a reduction in the risk of all complications in Group $2-1$ (2.0\%) compared with Group $1-9(17.7 \%)$ by $90.5 \%-\mathrm{OR}=0.095(0.002-0.75), \mathrm{p}=0.009$.

\section{Discussion}

As you can see, the immediate results of patients from groups 1 and group 2 were not significantly different and were comparable.

Long-term results in patients of groups 1 and 2 were different. In particular, in patients of group 1 chronic inguinal pain was in 4 (7.9\%), compared with the absence of chronic pain in patients of group 2 , i. e. there was a tendency to significantly improve the clinical outcome. The best results in the long-term postoperative period of 6-36 months were obtained in patients of the 2nd group, to whom were provided the improved TAPP technique, in comparison with the 1st group, to whom were performed the traditional TAPP technique. The absence of chronic postoperative pain in patients of the 2 nd group, after the improved TAPP technique, is explained by the fact, that with this technique, the fixation of the mesh along the lateral edges, where the branches of the femoral nerve pass, was performed with glue, what excluded its damage. The mechanical stapler fixation of the mesh with the traditional TAPP technique is most likely caused trauma to the branches of the femoral nerve, which leads to chronic postoperative pain.

Regarding repeated recurrences of inguinal hernias, there is a difference among patients of groups 1 and $2(1-5(9.8 \%), 2-1(2 \%))$, with a tendency to improve the clinical effect of improved TAPP. In observation of 5 patients with recurrent inguinal hernias that arose after the traditional TAPP technique in the 1st group of patients, with ultrasound diagnostics was found that in 4 cases 
a hernial defect was found along the upper edge of the mesh, which indicates the insufficiency of its overlap with the mesh and that it caused relapses. The improved TAPP technique, which was performed in the 2nd group, significantly reduced the probability of recurrent inguinal hernia, as long as it involves wider overlapping with a mesh of a larger size $(15 \times 15 \mathrm{~cm})$, of the upper edge of the hernia defect.

In 1 patient with a recurrent inguinal hernia after the traditional TAPP technique, the ultrasound diagnostics revealed a defect along the lower edge of the mesh, confirming the likelihood of flexion of the mesh because it does not fix on the lower edge. During the performance of the improved TAPP technique, the probability of relapse decreases, as far as the mesh on the lower edge is fixed with glue.

In 1 patient with a recurrent inguinal hernia after improved TAPP technique, an ultrasound scan revealed a recurrence along the upper edge of the mesh; the mesh was, most likely, migrated as a result of excessive physical exertion during the early postoperative period.

Thus, the results of the traditional and improved TAPP techniques confirm the higher efficiency of the improved technique. The frequency of the chronic inguinal pain after using the traditional TAPP technique is $7.9 \%$ and was absent after the performed improved TAPP technique. The frequency of the re-recurrence of inguinal hernia decreased to $2 \%$ when performed an improved TAPP technique, versus $9.8 \%$ with a traditional TAPP technique.

Study limitations. The study did not include patients with multiple recurrences of hernia, with inguinal-cellular recurrences of large hernias, intractable recurrent inguinal hernias, recurrences of inguinal hernias with concomitant decompensated cardiovascular and respiratory diseases, in portal hypertension cases.

Further research prospects. The prospect of the study is to study the laparoscopic IPOM and open method Stoppa effectiveness [19] in recurrent inguinal hernias and the study of the use of laparoscopic IPOM in recurrences of inguinal hernias after TAP with adhesive cover composite mesh using a [20].

\section{Conclusion}

Improved TAPP for recurrent inguinal hernias after Liechtenstein's surgery due to wider mobilization of parietal peritoneum on the upper edge of the defect, the use of mesh size and additional fixation with glue on the lower lateral edge, which does not cause trauma to the axillary nerves closes defects in the area of medial and lateral inguinal fossae, and reduces the probability of chronic inguinal pain from $7.9 \%$ in traditional TAPP to its absence in advanced TAPP, and reduces the recurrence of inguinal hernia from $9.8 \%$ in traditional to $2 \%$ in the advanced method TAPP.

\section{Conflict of interest}

The authors declare that they have no conflicts of interest.

\section{References}

[1] Simons, M. P., Aufenacker, T., Bay-Nielsen, M., Bouillot, J. L., Campanelli, G., Conze, J. et. al. (2009). European Hernia Society guidelines on the treatment of inguinal hernia in adult patients. Hernia, 13 (4), 343-403. doi: http://doi.org/10.1007/ s10029-009-0529-7

[2] Bhangu, A., Singh, P., Pinkney, T., Blazeby, J. M. (2014). A detailed analysis of outcome reporting from randomised controlled trials and meta-analyses of inguinal hernia repair. Hernia, 19 (1), 65-75. doi: http://doi.org/10.1007/s10029-014-1299-4

[3] Gopal, S. V., Warrier, A. (2013). Recurrence after groin hernia repair-revisited. International Journal of Surgery, 11 (5), $374-$ 377. doi: http://doi.org/10.1016/j.ijsu.2013.03.012

[4] The Danish hernia database (DHDB). Available at: http://www.danishhealthdata.com

[5] Eklund, A., Rudberg, C., Leijonmarck, C.-E., Rasmussen, I., Spangen, L., Wickbom, G. et. al. (2007). Recurrent inguinal hernia: randomized multicenter trial comparing laparoscopic and Lichtenstein repair. Surgical Endoscopy, 21 (4), $634-640$. doi: http://doi.org/10.1007/s00464-006-9163-y

[6] Itani, K. M. F., Fitzgibbons, R., Awad, S. S., Duh, Q.-Y., Ferzli, G. S. (2009). Management of Recurrent Inguinal Hernias. Journal of the American College of Surgeons, 209 (5), 653-658. doi: http://doi.org/10.1016/j.jamcollsurg.2009.07.015 
[7] Bisgaard, T., Bay-Nielsen, M., Kehlet, H. (2008). Re-recurrence After Operation for Recurrent Inguinal Hernia. A Nationwide 8-Year Follow-up Study on the Role of Type of Repair. Annals of Surgery, 247 (4), 707-711. doi: http://doi.org/10.1097/ sla.0b013e31816b18e3

[8] Swedish Hernia Register. Available at: http://www.svensktbrackregister.se

[9] Lee, S. S., Jung, H. J., Park, B. S., Son, G. M., Cho, Y. H. (2016). Surgical Aspects of Recurrent Inguinal Hernia in Adults. The American Surgeon, 82 (11), 1063-1067. doi: http://doi.org/10.1177/000313481608201120

[10] Alani, A., Duffy, F., O'Dwyer, P. J. (2006). Laparoscopic or open preperitoneal repair in the management of recurrent groin hernias. Hernia, 10 (2), 156-158. doi: http://doi.org/10.1007/s10029-005-0052-4

[11] Feleshtynskyi, Y. P., Shtaier, A. A., Yosypenko, M. O. (2019). The transabdominal preperitoneal aloplasty optimization for recurrent inguinal hernias after liechtenstein surgery. Surgery of Ukraine, 2, 30-33. doi: http://doi.org/10.30978/su2019-2-30

[12] Haapaniemi, S., Nilsson, E. (2002). Recurrence and Pain Three Years after Groin Hernia Repair. Validation of Postal Questionnaire and Selective Physical Examination as a Method of Follow-up. The European Journal of Surgery, 168 (1), $22-28$. doi: http://doi.org/10.1080/110241502317307535

[13] Campanelli, G., Pettinari, D., Nicolosi, F. M., Cavalli, M., Avesani, E. C. (2006). Inguinal hernia recurrence: classification and approach. Hernia, 10 (2), 159-161. doi: http://doi.org/10.1007/s10029-005-0053-3

[14] Feleshtinskyi, Ya. P., Shtaier, A. A. (2018). Pat. No. 129926 UA. Method of Transabdominal Preperitoneal Aloplasty in Recurrent Inguinal Hernia. MPK: A61B 17/00. No. u201803186. declareted: 27.03.2018; published: 26.11.2018. Bul. No. 22.

[15] Ceccarelli, G., Casciola, L., Pisanelli, M. C., Bartoli, A., Zitti, L. D., Spaziani, A. et. al. (2007). Comparing fibrin sealant with staples for mesh fixation in laparoscopic transabdominal hernia repair: a case control-study. Surgical Endoscopy, 22 (3), 668-673. doi: http://doi.org/10.1007/s00464-007-9458-7

[16] Novik, B., Hagedorn, S., Mörk, U.-B., Dahlin, K., Skullman, S., Dalenbäck, J. (2006). Fibrin glue for securing the mesh in laparoscopic totally extraperitoneal inguinal hernia repair: a study with a 40-month prospective follow-up period. Surgical Endoscopy, 20 (3), 462-467. doi: http://doi.org/10.1007/s00464-005-0391-3

[17] Alfieri, S., Amid, P. K., Campanelli, G., Izard, G., Kehlet, H., Wijsmuller, A. R. et. al. (2011). International guidelines for prevention and management of post-operative chronic pain following inguinal hernia surgery. Hernia, 15 (3), $239-249$. doi: http://doi.org/10.1007/s10029-011-0798-9

[18] Fränneby, U., Gunnarsson, U., Andersson, M., Heuman, R., Nordin, P., Nyrén, O., Sandblom, G. (2007). Validation of an Inguinal Pain Questionnaire for assessment of chronic pain after groin hernia repair. British Journal of Surgery, 95 (4), $488-493$. doi: http://doi.org/10.1002/bjs.6014

[19] Stoppa, R. E. (1989). The treatment of complicated groin and incisional hernias. World Journal of Surgery, 13 (5), $545-554$. doi: http://doi.org/10.1007/bf01658869

[20] Catani, M., De Milito, R., Materia, A., Chiaretti, M., Spaziani, E., Manili, G., Simi, M. (2003). Laparoscopic Inguinal Hernia Repair "IPOM” With Dual-Mesh. Annali Italiani di Chirurgia, 74 (1), 53-60. 